\title{
Current Collection to and Plasma Interaction with Femtosatellite- and CubeSat-Scale Electrodynamic Tether Subsystems
}

\author{
Jesse K. McTernan ${ }^{*}$ and Sven G. Bilén ${ }^{\dagger}$ \\ The Pennsylvania State University, University Park, Pennsylvania 16802, USA \\ Iverson C. Bell, $\mathrm{III}^{\ddagger}$ and Brian E. Gilchrist ${ }^{\S}$ \\ The University of Michigan, Ann Arbor, Michigan 48109, USA
}

\begin{abstract}
The efficacy of an electrodynamic tether system in generating a propulsive force or harvesting energy is limited, in part, by its ability to collect current from and emit current to the surrounding ambient plasma. This process is facilitated by active and passive electron emitters. Laboratory experiments were designed and conducted in a ground-based vacuum chamber to investigate the current-voltage characteristics of potential devices within the plume of a plasma source capable of producing ion and electron temperatures and densities similar to that found in low Earth orbit. We achieved active electron emission using a tungsten filament and lanthanum hexaboride crystal (approximately milliamps and microamps, respectively). We achieved a passive plasma contact by biasing a planar surface coated in a thin layer $(15 \Omega / \mathrm{sq})$ of indium tin oxide, which yielded current levels comparable to that of alodined aluminum. We also developed laboratory experiments designed to validate certain assumptions made about the behavior of ultra-small electron collecting interfaces for femtosatellites using short electrodynamic tethers.
\end{abstract}

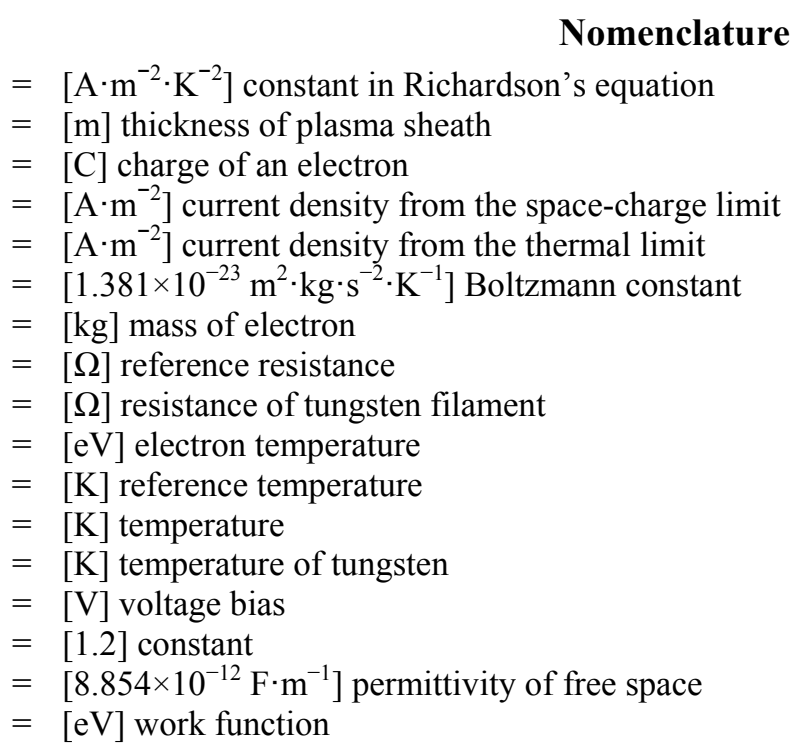

\footnotetext{
* Graduate Student Research Assistant, Aerospace Engineering, 229 Hammond Bldg., AIAA Student Member, jkm249@psu.edu.

$\dagger$ Associate Professor, Engineering Design, Electrical Engineering, and Aerospace Engineering, 213 Hammond Bldg., AIAA Associate Fellow.

* Ph.D. Candidate, Electrical Engineering, 1301 Beal Ave., AIAA Student Member.

$\S$ Professor, Electrical Engineering and Computer Science, 1301 Beal Ave., AIAA Associate Fellow.
} 


\section{Introduction}

$\mathrm{T}$ he efficacy of an electrodynamic tether (EDT) system to generate a propulsive force or to harvest energy is limited, in part, by its ability to collect current from and emit current to the surrounding magnetoplasma. ${ }^{1}$ Previous research has demonstrated that the efficiency of the tether-plasma interface for current collection and/or emission is typically the limiting factor to the overall system efficiency. ${ }^{2}$ However, the theoretical models used to capture the plasma physics and surface interactions present uncertainties. ${ }^{3}$ Furthermore, for the tether current levels needed for CubeSat- $(<10 \mathrm{~kg})$ and especially femtosatellite-class $(<100 \mathrm{~g})$ systems, it may be difficult to make simplifying approximations such as the assumption of a thin or thick current collecting sheath. Hence, experiments are necessary in order to properly characterize "contacting" devices - particularly at this scale.

The environment around an EDT or any spacecraft in low Earth orbit (LEO) may be described as a low-density cold (magneto-)plasma. Electron temperatures are on the order of $0.1-0.3 \mathrm{eV}$, ion temperatures are typically similar to the surrounding electrons, but because orbital velocities are faster than ion thermal velocities (mesosonic), relative to the spacecraft, ion motion is dominated by an apparent beam-like velocity that is on the order of $5 \mathrm{eV}$, and plasma densities are on the order of $10^{10}-10^{13} \mathrm{~m}^{-3}$ (with localized regions where the density can drop even lower, e.g. $10^{9}$ $\mathrm{m}^{-3}$ ) In general, past EDT experiments indicate that the LEO plasma acts as a low impedance path to "close the EDT circuit," but at the interface with the tether ends (e.g., spacecraft collecting surfaces or contactors) and any exposed tether conductor, an electrical "contact potential" region is established. An efficient electrical connection is essential to maximize current flow and thus propulsive thrust (e.g., $\mathrm{N} / \mathrm{kW}$ ).

This paper seeks to discuss efforts to better quantify EDT electrical end effects of concern for femtosatellite and Cubesat sized systems. The paper is divided into three sections; each is focused on a specific interface between tether end and the surrounding plasma. Section II presents electron and ion collection results from experiments with indium tin oxide-coated glass, which is intended to serve the dual purposes of covering solar panels as well as providing a plasma "contact". Section III concerns experimental results from active electron emission devices (thermionic cathode and lanthanum hexaboride) immersed in a LEO-like plasma. Section IV describes an experimental setup and feasibility study for ultra-small-scale ("femto-" and even "pico-" satellites) EDT systems that will utilize unique methods and geometries to interface the surrounding plasma.

\section{Indium Tin Oxide Experiment}

Indium tin oxide (ITO) is a mixture of indium (III) oxide $\left(\mathrm{In}_{2} \mathrm{O}_{3}\right)$ and tin (IV) oxide $\left(\mathrm{SnO}_{2}\right)$. When deposited as a thin film it has the characteristics of being optically transparent and electrically conductive. The thickness of the film governs the tradeoff between transparency and conductivity. ITO is widely used in LCDs, EMI shielding, touch screens, and other applications.

For small satellites (such as CubeSats), where outer surface area is at a premium, electrical power is at a premium, and simplicity is highly valued, solar panels are often mounted over much of the outer surfaces instead of being extended from the craft on deployable panels as in larger spacecraft. However, this outer surface area is also important for facilitating effiecient current collection. ITO-coated glass offers the potential advantage that it can be used to simultaneously create a conductive collecting surface on the solar panels without signicantly degrading the solar energy collecting function.

A thin film's conductivity or "sheet resistance" is often given in ohms per square $(\Omega / \mathrm{sq}$ or $\Omega / \square)$, which is the transverse resistance of a square surface patch. The sheet resistance is the resistivity of the film divided by the thickness of the film. The sheet resistance of our test article was $15 \pm 5 \Omega / \square$.



Figure 1. From left to right: aluminum test article, Langmuir probe, $\mathrm{LaB}_{6}$ crystal, and ITO test article. Plasma source in the background.

\section{A. Experimental Setup and Methods}

The experimental system used at The Pennsylvania State University is comprised of a vacuum chamber, plasma source, Langmuir probe, test articles or electron emitters, and software for measurement control and analysis. This 
description of the experimental setup applies to experiments reported in Sections II and III. Refer to Section IV for the experimental setup located at The University of Michigan.

\section{Vacuum Chamber}

The cylindrical vacuum chamber (Lesker Company) is approximately 40 inches $(101.6 \mathrm{~cm})$ in length and 35 inches $(88.9 \mathrm{~cm}$ ) in diameter (Fig. 1). Taking into account the feed-through ports, windows, and internal structures, the volume is approximately $24.4 \mathrm{ft}^{3}\left(0.69 \mathrm{~m}^{3}\right)$. A CTI-Cryogenics Cryo-Torr 7 high-vacuum pump can produce a base pressure of $2.2 \times 10^{-5}$ Torr. Two viewports allow visual confirmation of the creation of a plasma inside the plasma source. Refer to Ref. 4 for more details.

\section{Plasma Source}

Located in the vacuum chamber is a magnetic filter plasma source acquired from Colorado State University (Fig. 1). The source can simultaneously produce low temperature electrons $(0.1$ to $0.5 \mathrm{eV})$ and streaming ions $(5$ to $10 \mathrm{eV}){ }^{5}$ These conditions are similar to the conditions experienced by a spacecraft in low Earth orbit, where electrons collide with the surfaces due to their thermal velocity (on the order of $300 \mathrm{~km} / \mathrm{s}$ ) and ions stream to spacecraft surfaces due to the velocity of the spacecraft (approximately $7.5 \mathrm{~km} / \mathrm{s}$ ).

The gas feed system consists of an ultra-high purity regulator (Harris), 0-10 sccm mass flow controller (MKS), and a flow

Table 1. Plasma parameters $0.5 \mathrm{~m}$ down-stream from the source.

\begin{tabular}{|l|c|c|}
\hline Parameter & Value & Units \\
\hline Electron density & $6 \times 10^{11}$ & $\mathrm{~m}^{-3}$ \\
\hline Ion density & $2 \times 10^{12}$ & $\mathrm{~m}^{-3}$ \\
\hline Electron temperature & 0.10 & $\mathrm{eV}$ \\
\hline Plasma potential & 0.50 & $\mathrm{~V}$ \\
\hline Floating potential & 0.02 & $\mathrm{~V}$ \\
\hline Debye length & 3 & $\mathrm{~mm}$ \\
\hline
\end{tabular}
controller bypass that creates a low-conductance path from the vacuum chamber to the regulator. We currently use argon (99.999\% pure) as the ionizing gas. The flow rate for all experiments was $2.5 \mathrm{sccm}$ (except for Section III.B), which created a background pressure of $1.2 \times 10^{-4}$ Torr. This corresponds to atmospheric pressure at about $110 \mathrm{~km}$ altitude. The mean free path of the argon charge-exchange ions is approximately $0.5 \mathrm{~m}$ for these conditions.

Langmuir probe analysis was used to determine the plasma properties at the location of the experiments, which was approximately $0.5 \mathrm{~m}$ downstream of the plasma source (Table 1). Note that the conditions of the experiment correspond well to the ambient conditions of LEO except that no measurements of the ion temperature were made.

\section{Measurement and Analysis Software}

Current-vs.-voltage measurements were made with a Keithley 2410 SourceMeter controlled with a plasma analysis software suite developed inhouse. The software (a.k.a. "LP Suite") allows for automatic and manual analyses of I-V sweeps to determine parameters such as density and temperature of the charged particles, floating potential of the test piece or probe, plasma potential, Debye length, and other parameters.

Initial experiments revealed a significant amount of contamination of our probe and our reference test article as revealed by a hysteresis in voltage sweeps (Fig. 2). Contamination can be modeled as a parallel $\mathrm{RC}$ circuit placed between the surface of the conducting surface and the plasma. ${ }^{6}$ We developed a pulsed-voltage sweep based on the work of Ref. 7 that allows the capacitive component of the contamination to "discharge" between pulses, thus preventing the accumulation of unwanted charge that can become embedded in the contamination while voltage is

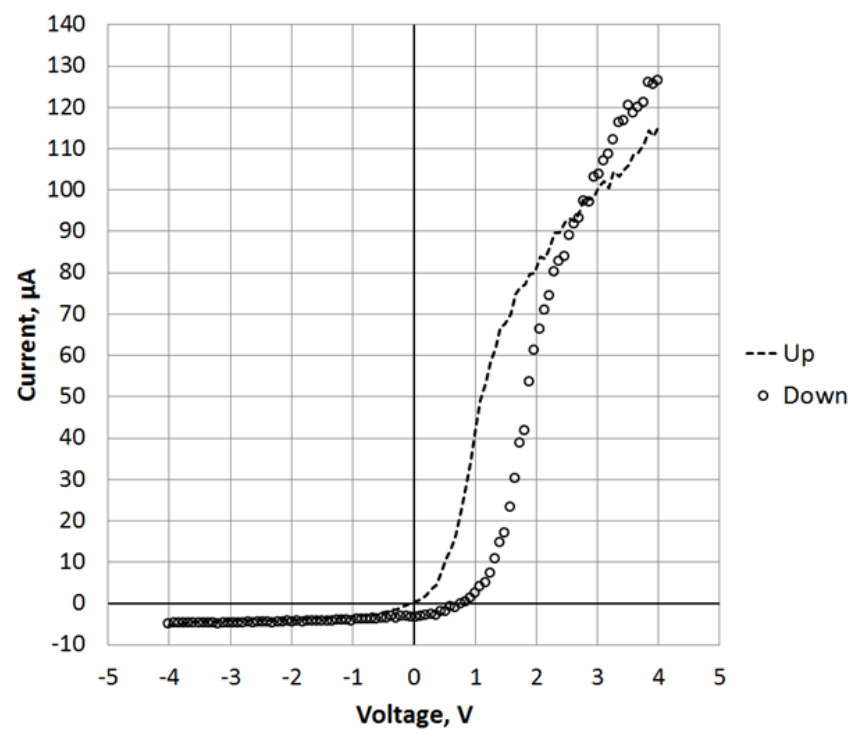

Figure 2. Hysteresis in the $I-V$ sweep of the aluminum test article indicates the presence of contamination.

applied. Using this pulsed-voltage technique, I-V sweeps had very little hysteresis giving us confidence that the sweep profiles were unaffected by contamination. 


\section{Langmuir Probe}

In order to measure plasma parameters, a cylindrical Langmuir probe was used that was previously developed at Penn State for operation on sounding rockets and small satellites. ${ }^{8}$ The collector section of the probe is $1 / 8$ inch $(6.13$ $\mathrm{mm})$ in diameter and 2 inches $(50.8 \mathrm{~mm})$ long (Fig. 3). The dimensions were chosen to ensure that the probe operates in the orbital-motionlimited (OML) regime and collects a readily

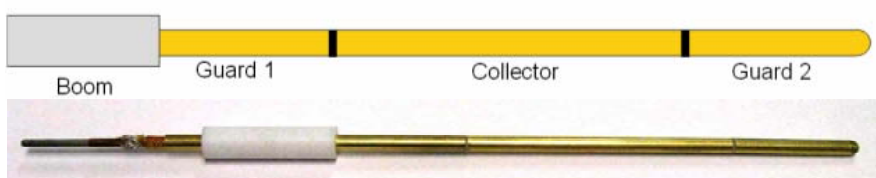

Figure 3. Langmuir probe designed at Penn State and used to measure the plasma characteristics in our test facility. measureable current. Two guard regions on either side of the collector mitigate fringing fields. The guards are held at the same potential as the collector. Each guard is approximately 1 inch $(25.4 \mathrm{~mm})$ long, with Guard 2 including a spherical tip. Teflon spacers of $0.01 \mathrm{inch}(0.25 \mathrm{~mm})$ are used to isolate the collector from the guards.

The probe is cleaned by applying a high positive and negative voltage (from -150 to $+150 \mathrm{~V}$ ) while immersed in the plasma. Incoming energetic charged particles collide with surface contaminates, which then dislodge from the surface. Cleaning by heating this probe is not possible. However, residual contamination remained and was accounted for using the technique mentioned in Section II.A.3.

\section{ITO and Reference Test Article}

The ITO and reference test articles were designed to perform as an ideal planar collecting surface. The test articles (collectors) were surrounded by a guard region that was held at the same electric potential as the collector section to mitigate fringing fields. The gap between the collector and guard was $0.5 \mathrm{~mm}$. The size of the center test article $(10 \times 10 \mathrm{~cm})$ conformed to the size restrictions of a single panel of a $1 \mathrm{U}$ CubeSat (Fig. 4).

Since the ITO film is deposited on a non-conductive substrate (glass), we laser etched the gap separating the collector from the guard. Therefore, both the test article and guard were on the same substrate for support, but were electrically isolated. Small holes drilled through the collector allowed a connection to the test article using wires that extended behind the article to the chamber feedthrough port.

The reference test article was fabricated using aluminum with a chromate-converted surface. This process, which is sometimes referred to as alodine, gold chromate, or iridite, protects the aluminum from corrosion and oxidation. We used an identically-sized $10 \times 10 \mathrm{~cm}$ square (Fig. 4) of aluminum alloy 6061 with a chromate conversion according to MIL-DTL-5541F Class 3 Type II. According to the specification, Class 3 coatings are qualified to have a resistance not greater than $5 \mathrm{~m} \Omega$ per square inch.

The test articles and Langmuir probe were positioned coplanar to each other and perpendicular to the axis of the

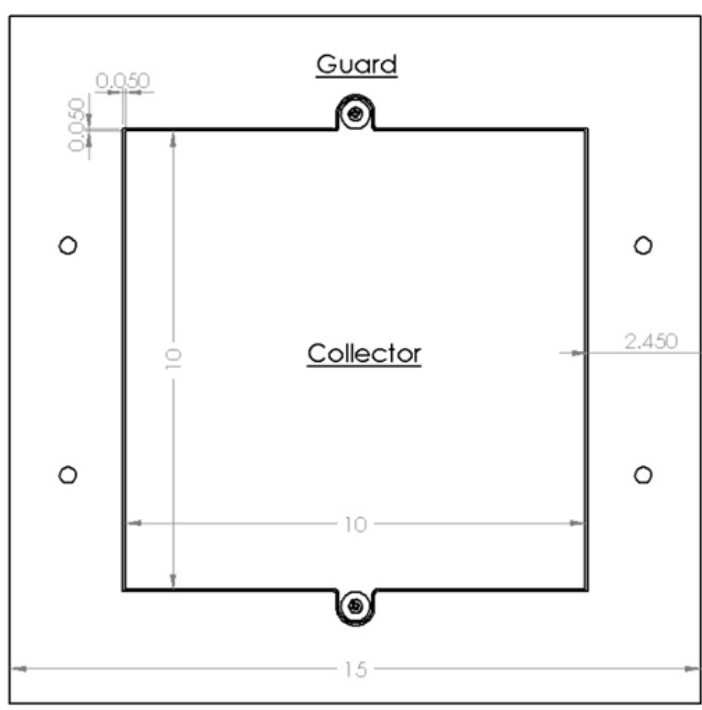

Figure 4. Dimensions for the ITO test article and aluminum reference $(\mathrm{cm})$. There is a $0.5-\mathrm{mm}$ gap between the collector and guard. Two screws make the connection between the surface and the measurement equipment.

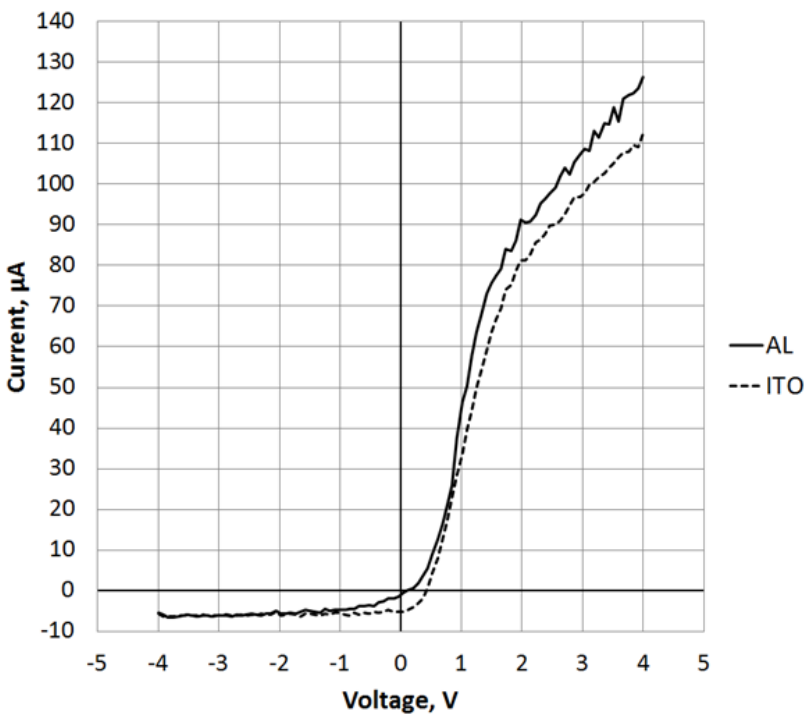

Figure 5. Comparison between I-V sweeps of the ITO test article and the aluminum reference shows order of magnitude similarity to current flow to and from the surfaces.

4

American Institute of Aeronautics and Astronautics 
source located $0.5 \mathrm{~m}$ away. The distance between the test articles and the Langmuir probe was approximately 3.5 in $(8.9 \mathrm{~cm})$, which was tens of Debye lengths of separation. Thus, no interference from adjacent experiments was expected. We have yet to determine the spatial gradients of the plasma plume, which would indicate if the test articles were in the same plasma environment as the Langmuir probe. Regardless, the test articles were placed symmetrically on either side of the probe and equidistant from the source so a comparison between the two is possible (Fig. 1).

\section{B. Results}

Comparison between the I-V sweeps of the ITO test article and the aluminum reference shows that for the tested voltage range, an ITO film has the capability of performing nearly equally as well (order of magnitude) as an aluminum surface in terms of current flow to or from the surface (Fig. 5). The trend in the figure seems to indicate that this near equality may not hold at higher current or voltage values. A larger voltage range and other conductors, such as gold plating, will be examined in future experiments.

The I-V sweeps reveal some differences in the performance of the ITO compared to aluminum. In the transition region from ion saturation to electron retardation, the ITO exhibits a sharper "knee" compared to the aluminum. Also, the general shape of the electron retardation region differs. The implications of these differences, if any, will be examined in future research.

\section{Active Electron Emission Experiments}

The experiment described in the previous section was designed to investigate passive electron and ion flow to a surface. Depending on the capability of a spacecraft, it may be possible to use active charge emission (or collection), which essentially requires some amount of energy to be expended to facilitate the current. The results of these experiments will be used in our efforts to create efficient plasma interfaces for small spacecraft.

The experimental setup for active electron emission was the same as the setup described in Section II except for the additional components needed to supply power to the emitters.

\section{A. Thermionic Cathode}

A thermionic cathode works on the principle of thermionic emission (i.e., a conductor emitting electrons when heated). The amount of current emitted depends on the temperature and is given by the Richardson's equation, ${ }^{9}$ i.e.,

$$
j_{\text {therm }}=A_{R} T^{2} \exp \left(\frac{-\psi}{k T}\right),
$$

where $j_{\text {therm }}$ is the current density emitted from the conductor, $A_{R}$ is a constant $\left(0.6 \mathrm{~A} \cdot \mathrm{m}^{-2} \cdot \mathrm{K}^{-2}\right.$ for tungsten), $T$ is the temperature, $\psi$ is the work function of the conductor, and $k$ is the Boltzmann constant. As electrons are emitted, the surrounding space becomes charged and it becomes increasingly more difficult to introduce more electrons reaching a point where no more electron charge can be emitted. This point is called the space charge limit $^{10,11}$ and is given by

$$
j_{\mathrm{SCL}}=\frac{4 \varepsilon_{0}}{9} \sqrt{\frac{2 e}{m_{e}}}\left(\frac{V^{3 / 2}}{d^{2}}\right),
$$

where $j_{\mathrm{SCL}}$ is the current density, $\varepsilon_{0}$ is the permittivity of free space, $e$ is the charge on an electron, $m_{e}$ is the mass of an electron, $V$ is the voltage of the anode with respect to the cathode, and $d$ is the distance between the anode and the cathode.* A thermionic device will be limited either thermally or by the space charge. If the surrounding environment has the ability to extract all of the heated electrons, then it is limited by the temperature (i.e., thermal limit). If the thermionic process produces more electrons than the surrounding space can extract, then it is limited by the space charge (i.e., space charge limit).

The advantage of a thermionic emitter is its potential simplicity relative to other electron emission technologies (e.g., plasma contactors, electron field emitters). However, the power requirements for the required heater and to overcome space charge limited flow can be significant depending on required emitted current levels. Similarly, robustness of the heated filament in the space and spacecraft near envirionment must also be considered.

\footnotetext{
** In the context of active emitters in the ionosphere, the anode refers to the plasma and the distance, $d$, is the distance of the plasma sheath.
} 


\section{Experimental Setup}

We spot welded a 0.011-in (0.2794-mm, 29-AWG) diameter, 1.9685-in $(5-\mathrm{cm})$ long tungsten wire to stainless steel rods that passed through ceramic supports (Fig. 6). The resistance of the filament at room temperature was $0.048 \Omega$. The stainless steel rods were attached perpendicularly to minimize electric fields from the rods or magnetic fields from the heating current. The assembly was supported by a FR4 rod, which is non-conductive and has low outgassing $(<1 \%$ total mass loss $)$.

We used a QuadTech 41021-80-60 programmable power supply to heat the filament and a Keithley 2410 SourceMeter to both bias the probe with respect to the plasma as well as measure the current flow through the filament into the plasma. The filament started to visibly glow around $2 \mathrm{~A}$. Measurements of thermionic emission were made with heater currents between 6.8 and $7.5 \mathrm{~A}$, which corresponded to tungsten temperatures ranging from approximately $2500 \mathrm{~K}$ to $2700 \mathrm{~K}$. Temperature, $T_{t}$, was inferred from the relationship,

$$
\frac{R_{t}}{R_{\text {ref }}}=\left(\frac{T_{t}}{T_{\text {ref }}}\right)^{\beta},
$$



Figure 6. Thermionic cathode experiment. Diameter and length of tungsten was $0.2794 \mathrm{~mm}$ and $5 \mathrm{~cm}$, respectively.

where $R_{t}$ is the resistance of the tungsten filament, $R_{\text {ref }}$ is the resistance of the filament at a reference temperature ( $T_{\text {ref }}$ ), and $\beta$ is a constant (1.2 for tungsten). ${ }^{12} R_{t}$ is calculated from the $\mathrm{I}-\mathrm{V}$ characteristic of the heater supply minus the residual resistance (from cables or connectors) measured prior to the experiment. Residual resistance was experimentally found to be constant through the heater current range.

\section{Thermionic Cathode Results}

The results of the thermionic cathode experiment are shown in Fig. 7. For each heater current (represented by the colored lines) the space charge and thermal limit are evident. When the filament was at a low bias with respect to the ambient plasma (ambient plasma potential was only about $0.5 \mathrm{~V}$ ), the voltage difference was not sufficient to extract all the heated electrons (space charge limit). As the voltage increased, the current increased until the thermal limit. At this point, an increase in voltage did not significantly extract more electrons. The space charge limit is seen in the lower left section of the contours in Fig. 7. The thermal limit is seen by the nearly flat plateaus toward the right.

For the setup tested, the power required to heat the filament (approximately $50 \mathrm{~W}$ ) exceeds the benefit in current, which is on the order of milliamps. Future experiments will use more energy efficient setups such as thinner filaments that heat up at lower power or accelerating grids placed in the

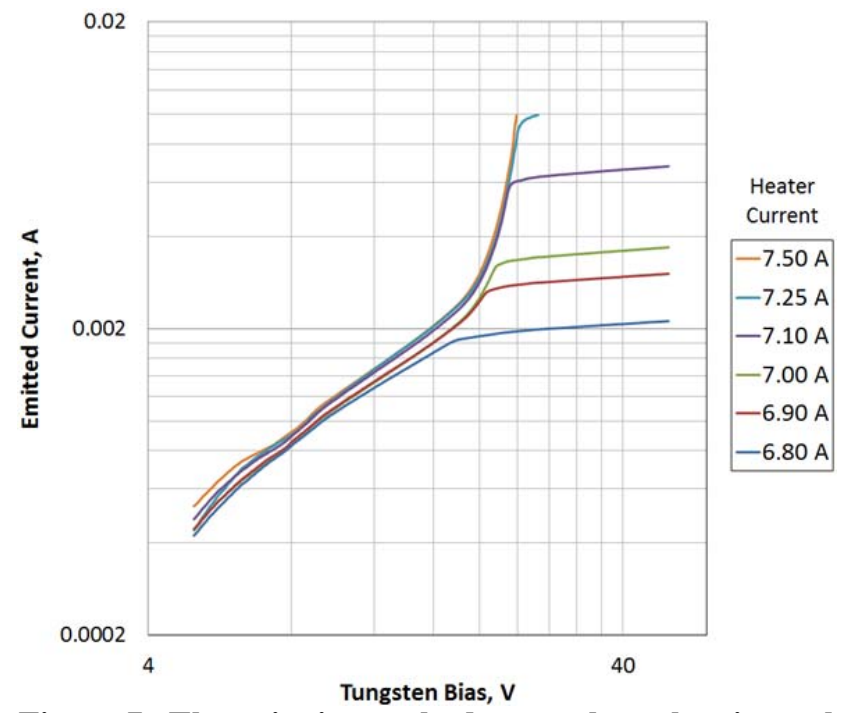

Figure 7. Thermionic cathode results showing the space-charge and thermionic limit. Milliamps of current was obtained. vicinity of the filament.

\section{B. Lanthanum Hexaboride $\left(\mathrm{LaB}_{6}\right)$}

Lanthanum hexaboride $\left(\mathrm{LaB}_{6}\right)$ crystals have a lower work function $(2.3 \mathrm{eV})^{13}$ than that of tungsten $(4.54 \mathrm{eV})$, which means that it takes less energy to extract electrons. We performed a similar experiment to that of the tungstenbased thermionic cathode (Section III.A), except that the cathode of interest in this experiment was lanthanum hexaboride. 


\section{Experimental Setup}

We used a Kimball Physics ES423E single crystal that has a $90^{\circ}$ cone and 15-micron microflat tip. The crystal was heated resistively by applying current through a carbon heater rod (Fig. 8.a) and was supported by a carbon ferrule (Fig. 8.b). The setup was biased negatively with respect to the ambient plasma so that the plasma would attract (and therefore extract) the heated electrons. A Keithley 2410 biased the setup as well as measured the emitted current.

\section{Results}

Results from the $\mathrm{LaB}_{6}$ experiment are presented in two sections: low power input and high power input. In this case, power refers to the amount of input energy expended to heat the crystal and excite electrons.

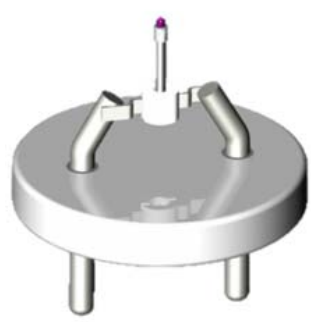

a.

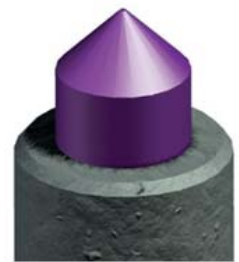

b.

Figure 8. $\mathrm{LaB}_{6}$ (a) test setup and (b) magnified view of the crystal. Images from Kimball Physics.

At low power $(0.17 \mathrm{~W})$, the crystal remained relative cool compared to its maximum heating capacity. ${ }^{\dagger \dagger}$ Although the limiting factor in this scenario was the temperature, we observed a moderate increase in current at high voltages. Small spacecraft would be power limited due to limited surface area for solar panels or limited volume for batteries; however small spacecraft equipped with EDT-systems have the potential to generate large voltage potentials $(>100 \mathrm{~V})$ with respect to the plasma. Therefore, the low-power scenario for small spacecraft is obtainable. Fig. 9 shows the current emitted from the test setup as measured by our biasing supply for the low-power scenario. Microamps of current flow into the plasma was obtained. The fact that there was some current flow without plasma indicates that there is a current sink elsewhere that needs to be investigated.

At higher heater power $(3.3 \mathrm{~W})$, the process of emitting electrons becomes limited by the space charge. As seen in our results in Fig. 10, microamps of current was obtainable at a bias voltage of a few volts. The transition from space charge limited to thermally limited occurred at a bias voltage of approximately $3 \mathrm{~V}$. Biasing the crystal further than this transition point produces only a slight increase in current. Therefore, if more current is needed the crystal will need to be heated more, larger in size, or a large bias $(>100 \mathrm{~V})$ will be needed. The contours in Fig. 10 demonstrate the change in emitted current at different flow rates (i.e., plasma densities).

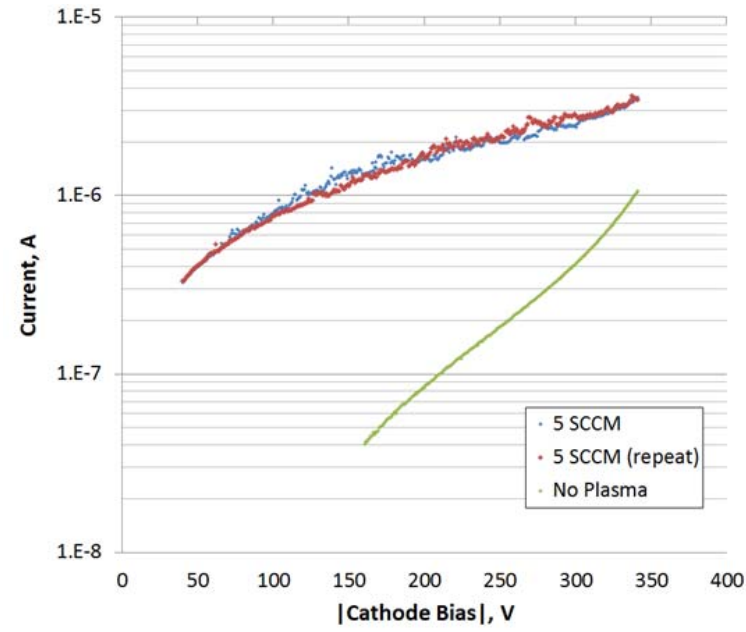

Figure 9. Current emitted from $\mathrm{LaB}_{6}$ crystal using $0.17 \mathrm{~W}$ of input power.

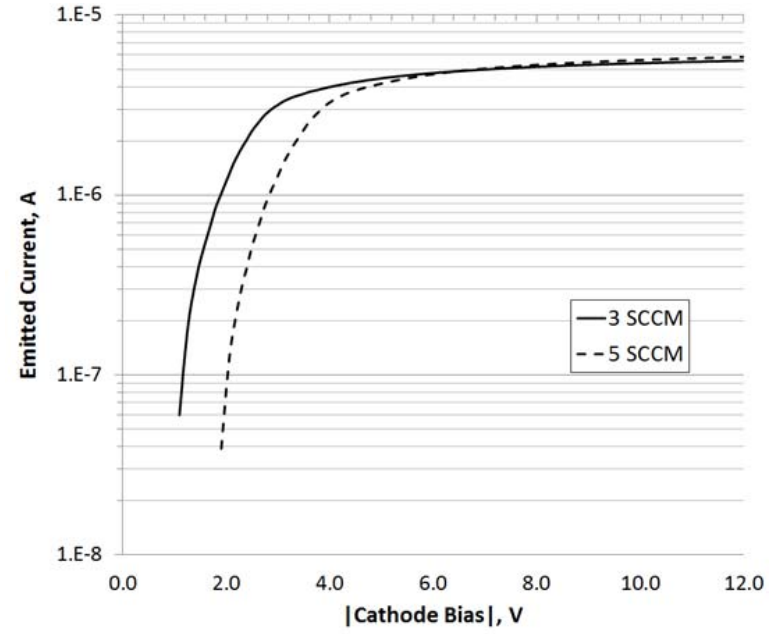

Figure 10. Current emitted from $\mathrm{LaB}_{6}$ crystal using $3.3 \mathrm{~W}$ of input power. Both the space-charge and thermally-limited regions are visible.

\footnotetext{
† Temperature values as a function heater current was provided by the supplier of the crystal.
} 


\section{Ultra Small-Scale Experiment and Feasibility Study}

\section{A. Introduction}

The picosatellite $(100 \mathrm{~g}-1 \mathrm{~kg})$ and femtosatellite $(<100 \mathrm{~g})$ architectures, often referred to as a "satellite-on-achip", "ChipSat", or "ultra-small satellite", are extremes in satellite miniaturization. The small size of ultra-small satellites allows them to be manufactured inexpensively and in large numbers while being boosted into orbit at a fraction of the cost of more massive and larger satellites. ${ }^{14}$ An entirely new class of simultaneous, multi-point sensing missions can be made possible if fleets of ChipSats are able to form reconfigurable, adaptable fleets. This capability, however, will require each satellite to have some degree of maneuverability and propulsion. The flat ChipSat wafer also has a short lifetime due to an inherently high area-to-mass ratio. Thus, propulsion will help unlock the full potential of the ultra-small satellite fleet concept.

The ability of miniaturized electrodynamic tethers to provide ultra-small satellites with propellantless propulsion and attitude stability has been explored in several trade studies. ${ }^{15-19}$ The system concept incorporates an insulated, conducting tether connecting a pair of nearly identical satellites that work together as a single element. Each satellite is equipped with a solar panel, power supply, and cold cathode electron emitter, and is capable of collecting electrons on the surface. The electrons flowing in the tether generate thrust needed for drag make-up and propulsion. Electrons are collected from the ambient plasma by the anode and emitted into the plasma by the cathode. In order to evaluate the feasibility of the ultra-small satellite EDT concept, it is important to understand how efficiently electron current can be collected by the anode and emitted by the cathode.

Although there are models that allow us to estimate the electron emission current from the cathode ${ }^{20}$ the authors have not identified models that predict the expected collected anode current given the likely non-standard geometries of the anode collecting endbody. A variety of simplifying assumptions were made in previous trade studies to facilitate estimating this current using simple geometry models. ${ }^{19}$ Our goal is to improve our anode current collection estimate by conducting ground-based plasma experiments that capture critical characteristics of the LEO environment.

\section{B. Plan for Picosatellite and Femtosatellite Current Collection Experiment}

One of our goals is to assess the feasibility of the EDT and dual pico-/femtosatellite concept. The system concept is capable of boosting and deboosting, but the magnitude of thrust is related to the EDT current, so it is critical to estimate the tether current in order to evaluate the feasibility of EDT propulsion. Here, we describe a ground-based current collection experiment that captures key characteristics of the satellite and its environment.

In our system concept, we assume that the pico- or femtosatellite outer surface can be coated in a transparent conductor such as ITO and biased above the plasma potential to collect electrons. Electron current collection is dependent on anode geometry, voltage relative to plasma potential, and ambient conditions. The possible cuboid shape of the pico- and femtosatellite, the relative motion of the plasma, and the presence of an ambient magnetic field add complexity to predicting collection current.

In previous trade studies, we made several simplifying assumptions to estimate current. Conventional current collection theory describes collection to geometries such as an infinite plate, infinitely long cylinder, and sphere. Our pico/femtosatellites, however, will likely be cuboids. The authors are not aware of any experiments in LEO that studied current collection to similar structures of the same scale in typical ionospheric conditions. We assumed that at high potentials $\left(V_{\text {probe }}>>k T_{e}\right.$ ), the pico/femtosat's I-V characteristic would be similar to the I-V characteristic of an equivalent sphere. Although this seems plausible for a small cube-shaped probe, it is less so for a thin plate. After assuming a spherical collector, we attempted to identify an appropriate model. There are a variety of models for spherical collectors, but it was unclear which model would most accurately estimate the collected current for a pico/femtosat in LEO. All of the models considered are described in Ref. 19.

We ultimately chose to be conservative and used an expression developed by Barjatya et al. ${ }^{21}$ The expression was empirically derived for extracting plasma parameters (i.e., electron temperature and density) using a $5-\mathrm{cm}$ radius gold spherical Langmuir probe located on the International Space Station. We wish to compare the experimentally measured current with this model and the OML and Parker-Murphy models. ${ }^{11,22}$

The experimental facility will be the cathode test facility (CTF) at University of Michigan's Plasmadynamics and Electric Propulsion Laboratory, which is a $2-\mathrm{m}$ (78.7 in) long, $60-\mathrm{cm}$ (23.6 in) diameter cylinder. The flowing plasma will be generated by a $\mathrm{LaB}_{6}$ hollow cathode, which simulates the relative velocity between the ionospheric plasma and the orbiting satellite. We will place the probes at a few different positions relative to the cathode exit plane. Our goal is for the probes to be in a location where the Debye length is between $0.1 \mathrm{~cm}$ and $1 \mathrm{~cm}$, which is roughly the Debye length range in LEO. Expecting the electron temperature to be a few eV downstream of the plume, we desire a plasma density between $1 \times 10^{12} \mathrm{~m}^{-3}$ and $1 \times 10^{14} \mathrm{~m}^{-3}$ to ensure that the Debye length will be in this 
range. In previous experiments using $\mathrm{CTF}$ and $\mathrm{LaB}_{6}$ cathode, a Langmuir probe positioned about $5 \mathrm{~cm}$ from the centerline of the cathode's exit plane and about $16 \mathrm{~cm}$ downstream from the cathode measured an electron temperature of about $5 \mathrm{eV}$ and electron density of about $4.2 \times 10^{17} \mathrm{~m}^{-3}$, so the probes will need to be farther downstream from the exit plane than this. ${ }^{23}$



a.

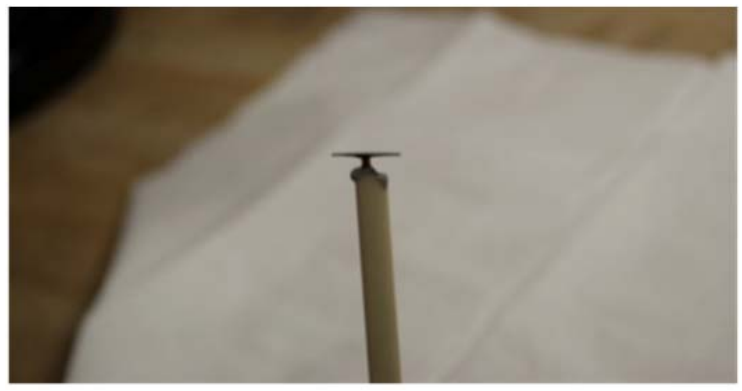

c.

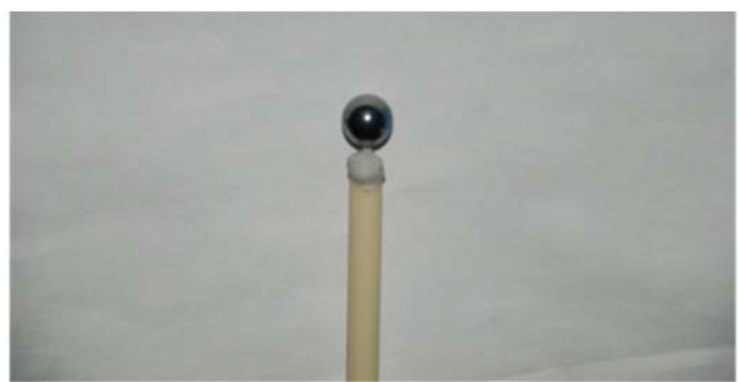

b.



d.

Figure 11. (a) Cylindrical (reference) probe; (b) Spherical probe; (c) Thin plate probe; (d) Two cylindrical probes separated by an angle.

We desire to record the $\mathrm{I}-\mathrm{V}$ characteristics of four probes. First, we would like to measure the $\mathrm{I}-\mathrm{V}$ characteristic of a probe that is roughly the same shape of a pico- or femtosat (cuboid) and size relative to the Debye length. In the previous iteration of the trade study, ${ }^{19}$ we assumed that the anode collects current like a sphere with an equivalent diameter equal to the satellite's longest edge, so measurements of a spherical probe with a diameter equal to the plate width will also be taken to evaluate this assumption. The I-V characteristics of a cylindrical probe will also be measured for comparison. Finally, two cylindrical probes offset by some angle will be studied. This configuration will be the simplest form of the many-spoked "pom-pon" or "hedgehog" collector described by Ref. 24. Multiple cylindrical probes may provide a means for efficient electron collection without significantly increasing the ram area and therefore drag of an ultra-small satellite. Probes are shown in Fig. 11a-d. Each probe tip is made from tungsten and connected to tungsten wire located inside an alumina tube.

The four probes will be placed on a rotary table. The rotary table allows the probes to be rotated between I-V sweeps so the sweep from each probe will be taken in the same location relative to the cathode exit plane. Each of the probe voltages considered for the experiment will be roughly scaled so that the voltage-to-electron temperature ratio is near what is expected in LEO. The voltage range will include a high resolution sweep in the ion saturation and electron retardation range as well as a sweep well into the electron saturation.

\section{Summary and Conclusion}

Using ground-based experiments we were able to test passive and active electron emission from various sources into a LEO-like plasma. For passive electron emission, we found that a thin layer of indium tin oxide-coated glass can facilitate current collection comparable to aluminum (order of magnitude) for the tested voltage range. This coated glass can also be used to cover solar panels, thus allowing a small spacecraft's limited surface area to serve a dual purpose.

Active electron emission experiments from heated tungsten demonstrated that our setup could emit current into the plasma (milliamps), but operated at input power levels that were too high (tens of watts) for practical use. Small spacecraft will be power limited. Therefore, a more efficient tungsten setup will be investigated. 
We also performed experiments with low-power electron emission using lanthanum hexaboride. Microamps of current was obtained using only $0.17 \mathrm{~W}$ of input power, but at high voltage. Some of the current was being sinked into unknown locations, which will be investigated in future work. Nonetheless, microamps of current higher than our no-plasma control current was observed. At higher power $(3.3 \mathrm{~W})$, microamps of current was obtained at much smaller voltages.

We developed and are preparing more ground-based experiments to investigate certain assumptions we made about ultra-small scale plasma interfaces. These assumptions were used in numerous feasibility studies focused on drag-makeup and maneuverability of pico- and femto-scaled satellites.

\section{Acknowledgments}

The authors thank J. Williams (Colorado State Univ.) for his contributions to our research through continuous technical and knowledgeable support on the plasma source, V. Getman (Penn State) and K. Trent (Univ. of Mich.) for laboratory support, and A. Soares for experimental support during summer 2012. We are grateful for funding from AFOSR via grant FA9550-09-1-0646.

\section{References}

${ }^{1}$ Cosmo, M.L., and Lorenzini, E.C., eds., Tethers In Space Handbook, 3rd Edition, NASA/SAO, 1997.

${ }^{2}$ McTernan, J.K., "Development of a Modeling Capability for Energy Harvesting Modules in Electrodynamic Tether Systems,” M.S. Thesis, Aerospace Engineering, Pennsylvania State Univ., University Park, PA, 2011.

${ }^{3}$ Gilchrist, B.E., et al., "The PROPEL Electrodynamic Tether Mission and Connecting to the Ionosphere," Spacecraft Charging and Technology Conference, Kitakyushu, Japan, May 15, 2012.

${ }^{4}$ McTernan, J.K., and Bilén, S.G., "System for Measuring Current-Voltage Characteristics of Collectors and Emitters for Small-Scale Electrodynamic Tether Systems," Spacecraft Charging and Technology Conference, Kitakyushu, Japan, May 15, 2012.

${ }^{5}$ Williams, J.D., Farnell, C.C., Shoemaker, P.B., Vaughn, J.A., and Schneider, T.A., "Ground-Based Simulations of Low Earth Orbit Plasma Conditions: Plasma Generation and Characterization," 8th Spacecraft Charging Technology Conference, NASA Marshall Space Flight Center, Huntsville AL, Oct. 20-23, 2003.

${ }^{6}$ Oyama, K., “A Systematic Investigation of Several Phenomena Associated with Contaminated Langmuir Probes,” Planet. Space Sci., Vol. 24, pp.183-190, 1976.

${ }^{7}$ Szuszczewicz, E.P., and Holmes, J.C., "Surface Contamination of Active Electrodes in Plasmas: Distortion of Conventional Langmuir Probe Measurements," J. Appl. Phys. 46, 5134, 1975.

${ }^{8}$ Escobar, A.C., "A Langmuir Probe Instrument for Research in the Terrestrial Ionosphere," M.S. Thesis, Electrical Engineering, Pennsylvania State University, University Park, PA, 2009.

${ }^{9}$ Richardson, O.W., The Emission of Electricity from Hot Bodies, $2^{\text {nd }}$ ed., Longmans, Green and Co., London, 1921.

${ }^{10}$ Langmuir, I., "The Effect of Space Charge and Initial Velocities on the Potential Distribution and Thermionic Current between Parallel Plane Electrodes," Physical Review, Vol. 21, No. 4, pp. 419-435, 1923.

${ }^{11}$ Mott-Smith, H.M., and Langmuir, I., "The Theory of Collectors in Gaseous Discharges," Phys. Rev., Vol. 28, pp. 727-763, 1926.

${ }^{12}$ Forsythe, W.E., and Worthing, A.G., "The Properties of Tungsten and the Characteristics of Tungsten Lamps," Astrophysical Journal, Vol. 61, p. 146, 1925.

${ }^{13}$ Yamauchi, H., Takagi, K., Yuito, I., and Kawabe U., "Work Function of LaB, ," Appl. Phys. Lett. Vol. 29, p. 638, 1976.

${ }^{14}$ Barnhart, D.J., Vladimirova, T., Baker, M., and Sweeting, M. N., "A Low-cost Femtosatellite to Enable Distributed Space Missions," Acta Astronautica, pp. 1123-1143, June 2009.

${ }^{15}$ Bell, I.C., "Electrodynamic Tethers for ChipSat and Nanospacecrafts." Poster, Spacecraft Charging and Technology Conference, Albuquerque, NM, Sept 23, 2010.

${ }^{16}$ Bell, I.C., Gilchrist, B., McTernan, J.K., Bilén, S.G., Voronka, N., Hoyt, R., and Peck, M., "The Potential of Miniature Electrodynamic Tethers to Enhance Capabilities of Femtosatellites," IEPC-2011-054, 32nd International Electric Propulsion Conference, Wiesbaden, Germany, Sept. 15, 2011.

${ }^{17}$ Bell, I.C., Gilchrist, B., McTernan, J.K., Bilén, S.G., Voronka, N., Hoyt, R., and Peck, M., "Enabling Ultra-small Sensor Spacecraft for the Space Environment using Small-Scale Electrodynamic Tethers," AIAA-2011-7322, AIAA SPACE 2011 Conference and Exposition, Long Beach, California, Sept. 27-29, 2011.

${ }^{18}$ Bell, I.C., Liaw, D., Gilchrist, B., McTernan, J.K., Bilén, S., Voronka, N., Hoyt, R., and Peck, M., "Electrodynamic Tethers for ChipSat and Nanospacecrafts," Spacecraft Charging and Technology Conference, Kitakyushu, Japan, May 15, 2012.

${ }^{19}$ Bell, I.C., Liaw, D., Gilchrist, B., McTernan, J.K., and Bilén, S., "Investigating the Use of Miniaturized Electrodynamic Tethers to Enhance the Capabilities of Femtosatellites and Other Ultra-small Satellites," AIAA/Utah State Small Satellite Conference, Logan, Utah, Aug. 16, 2011.

${ }^{20}$ Whaley, D., et al., "100 W Operation of a Cold Cathode TWT," IEEE Trans. Electron Devices, Vol. 56, No. 5, pp. 896905, May 2009. 
${ }^{21}$ Barjatya, A., Swenson, C.M., Thompson, D.C., and Wright, K.H., Jr.,"Invited Article: Data Analysis of the Floating Potential Measurement Unit aboard the International Space Station,”Rev. Sci. Intrum., Vol. 80, No. 4, 2009.

${ }^{22}$ Parker, L.W., and Murphy, B.L., "Potential Buildup on an Electron Emitting Ionospheric Satellite," J. Geophys. Res., Vol. 72, p. 1631, 1967.

${ }^{23}$ Trent, K.R., McDonald, M.S., Lobbia, R.B., Gallimore, A.D., "Time-resolved Langmuir Probing of a New Lanthanum Hexaboride $\left(\mathrm{LaB}_{6}\right)$ Hollow Cathode," IEPC-2011-245, 32nd International Electric Propulsion Conference, Wiesbaden, Germany, September 11-15, 2011.

${ }^{24}$ Voronka, N., Hoyt, R.P., Gilchrist, B.E., and Fuhrhop, K., "An Architecture of Modular Spacecraft with Integrated Structural Electrodynamic Propulsion (ISEP),” NIAC 7 the Annual Meeting, Tucson, AZ, October 18, 2006. 